\title{
Evolving robust policies for community energy system management
}

Supplementary Material

\author{
RUI P. CARDOSO, Imperial College London, UK \\ EMMA HART, Edinburgh Napier University, UK \\ JEREMY V. PITT, Imperial College London, UK
}

ACM Reference Format:

Rui P. Cardoso, Emma Hart, and Jeremy V. Pitt. 2019. Evolving robust policies for community energy system management: Supplementary Material. 1, 1 (April 2019), 12 pages. https://doi.org/10.1145/3321707.3321763

\section{MODEL}

In this work, we have modelled an energy system encompassing several CESs. While this model has been based on knowledge arising from some of the literature on CESs $([1,2,5,6,8,10])$, it is mostly intuitive. It abstracts a hypothetical energy system connecting several communities which both produce and consume energy and are able to trade it directly amongst themselves. We are not aware of an actual system of this kind existing or of any theories which can be applied directly to our model; our mental model is the only source of knowledge about the problem domain. This places our modelling approach within the System Dinamycs (SD) paradigm, created by Forrester [4]. It is important to note that we are not excessively concerned with the realism of our model, as it has been designed mostly for enabling the study of adaptation and evolution of operating policies.

In the model which we have constructed, energy is treated as a CPR and communities as agents. The communities are part of an energy system; they have energy demands and can generate energy from a number of renewable resources. Three sources of renewable energy have been considered, namely photovoltaic cells, wind turbines, and hydropower converters. The energy system can also generate and feed energy to compensate for any lack of self-generated power. Communities are located on a grid and have neighbours with which they are able to trade energy, using a simplified version of the Contract Net Protocol [9]; they are also able to trade energy with the central system. A time step corresponds to an hour in the simulation time. At each time step, the energy system uses the current operating policy to determine the mode of operation of the system for that time step, as explained in detail in section 2. Figure 1 summarises the domain model of the system.

As an attempt to create a flexible model, we have defined a number of input parameters required for instantiating it. These parameters are summarised in Table 1 . There are a certain number of communities connected to the energy system, given by num_communities. They are placed randomly on a grid, each community occupying a single cell and each cell being occupied, at most, by a single community. The size of the grid is given by parameters width and height. Each community is able to store energy up until a certain maximum amount,

Authors' addresses: Rui P. Cardoso, rui.cardoso@imperial.ac.uk, Dept. of Electrical and Electronic Engineering, Imperial College London, London, UK, SW7 2AZ; Emma Hart, e.hart@napier.ac.uk, School of Computing, Edinburgh Napier University, Edinburgh, UK, EH10 5DT; Jeremy V. Pitt, j.pitt@imperial.ac.uk, Dept. of Electrical and Electronic Engineering, Imperial College London, London, UK, SW7 2AZ.

Permission to make digital or hard copies of all or part of this work for personal or classroom use is granted without fee provided that copies are not made or distributed for profit or commercial advantage and that copies bear this notice and the full citation on the first page. Copyrights for components of this work owned by others than ACM must be honored. Abstracting with credit is permitted. To copy otherwise, or republish, to post on servers or to redistribute to lists, requires prior specific permission and/or a fee. Request permissions from permissions@acm.org.

(C) 2019 Association for Computing Machinery.

XXXX-XXXX/2019/4-ART \$15.00

https://doi.org/10.1145/3321707.3321763

, Vol. 1, No. 1, Article . Publication date: April 2019. 


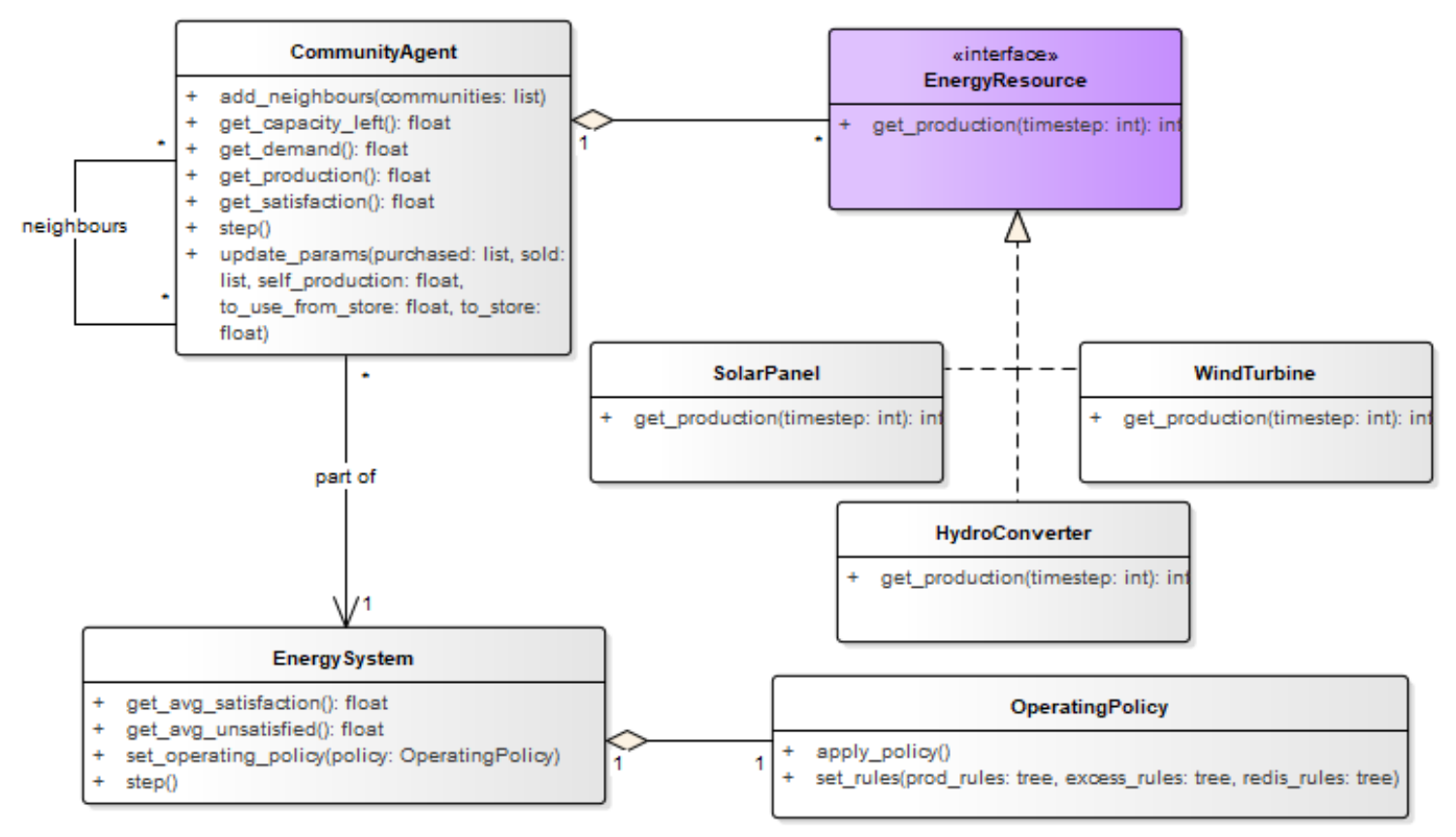

Fig. 1. Domain model of the energy system

given by its capacity; the capacity is a random positive integer, with min_capacity being its minimum value and maximum_capacity its maximum value. The cost of storing energy is drawn from a uniform distribution, such that the minimum possible cost is given by min_storage_cost and the maximum cost is given by max_storage_cost. The neighbours of a community are determined by the parameter neighbour_radius, which is a positive integer representing a number of cells; all communities within this number of cells from a certain community in the grid, including diagonals, are considered its neighbours. The notion of neighbour is important, as communities are only allowed to trade energy with their neighbours. The central system is able to flexibly produce energy at each time step if there is at least one community whose demands have not been satisfied; in this case, the unit cost of production is given by production_cost. As part of its operation, the system will purchase energy from the communities and then resell it. The parameter purchasing_price is the unit price for which the central system purchases energy and the parameter selling_price is the unit price for which it sells it. The parameter $w$ is used to update the satisfaction of each community, as in equation 7, and the parameters alpha and beta are used to calculate the system performance, as in equation 8 . The parameter $n_{-}$each_resource determines the number of resources of each kind (photovoltaic cells, wind turbines, and hydropower converters) that each community with resources possesses and the parameter perc_with_resources determines the proportion of communities which do have access to renewable energy resources. The parameter seed sets the random seed of the model, ensuring reproducible results. Leaving this parameter undefined causes the model to use sources of randomness offered by Python, the language in which it has been implemented. 
The communities are initialised with some renewable energy resources, whose energy production is simulated. The energy produced by each community is obtained at each time step by summing over the energy produced by each of their energy resources. Communities also demand a certain amount of energy at each time step. We have used intuitive notions for coming up with functions which return the energy produced by each resource and each community's demand at every time step. These functions are defined by a sum of radial basis functions [3]. Given a set of centres, $\left\{c_{i}\right\}_{i=1}^{N}$, a set of bandwidths, $\left\{h_{i}\right\}_{i=1}^{N}$, and a set of weights, $\left\{w_{i}\right\}_{i=1}^{N}$, the sum of radial basis functions at time step $t$ is given by:

$$
S\left(t \mid\left\{w_{i}\right\}_{i=1}^{N},\left\{c_{i}\right\}_{i=1}^{N},\left\{h_{i}\right\}_{i=1}^{N}\right)=\sum_{i=1}^{N} w_{i} \times \exp \left(t \bmod 24-c_{i}\right)^{2} / h_{i}^{2}
$$

The number of radial basis functions, $N$, used to define this sum is given by the model input parameter num_rbfs. The greater the value of num_rbfs, the more fine-grained the sum defined by equation 1, which enables more complex functions (more inflection points) to be modelled. The weights, centres, and bandwidths are set intuitively according to each energy resource and the expected demand from communities. For each type of energy resource and for the demand of each community, the centres are set to fixed values; the weights and bandwidths, on the other hand, are drawn from probability distributions, as described ahead. For solar panels, the centres are set and the weights are drawn from Gaussian distributions such that there is a maximum at around midday everyday. The bandwidths are each drawn from a uniform distribution. The energy produced by a solar panel at time step $t$ is then given by:

$$
\begin{aligned}
& x \sim \mathcal{U}(0,1) \\
& E_{\text {solar }}\left(t \mid Q=\left\{\left\{w_{i}\right\}_{i=1}^{N},\left\{c_{i}\right\}_{i=1}^{N},\left\{h_{i}\right\}_{i=1}^{N}\right\}\right) \\
& = \begin{cases}(1-\text { loss_cloudy }) \times \max (S(t \mid Q), 0) & \text { if } x \leq \text { perc_cloudy } \\
\max (S(t \mid Q), 0) & \text { otherwise }\end{cases}
\end{aligned}
$$

The value perc_cloudy is the probability that the weather is cloudy at any given time step and loss_cloudy is the decrease (a percentage) in energy produced by the solar panel when the weather is cloudy. They are both drawn from uniform distributions. For both wind turbines and hydropower converters, the centres are set with a fixed step and the weights are drawn from a Gaussian distribution and are independent from the time of the day. The bandwidths are drawn from uniform distributions, as well as a positive offset intended to ensure that the energy produced by these two kinds of resources is never 0 . At time step $t$, the energy produced by either a wind turbine or a hydropower converter is then given by:

$$
E_{\text {wind,hydro }}\left(t \mid Q=\left\{\left\{w_{i}\right\}_{i=1}^{N},\left\{c_{i}\right\}_{i=1}^{N},\left\{h_{i}\right\}_{i=1}^{N}\right\}\right)=\text { offset }+\max (S(t \mid Q), 0)
$$

When defining the function returning a community's demand, the weights are set, again intuitively, so that there is a peak in the early morning and in the evening, by drawing from Gaussian distributions. The demand of a community at time step $t$ is then given by:

$$
D\left(t \mid Q=\left\{\left\{w_{i}\right\}_{i=1}^{N},\left\{c_{i}\right\}_{i=1}^{N},\left\{h_{i}\right\}_{i=1}^{N}\right\}\right)=\max (S(t \mid Q), 0)
$$

We defined the sum of radial basis functions, given by equation 1 , by calculating the modulo 24 of the input time step $t$. This has enabled us to define some daily trends, such as the energy produced by solar panels peaking at around midday or the energy demands peaking in the early morning and in the evening. However, it also causes this function to repeat itself every 24 time steps. We address this by adjusting the weights according to another input parameter, noise. We start by generating a base set of weights, $\left\{w_{i}^{\prime}\right\}_{i=1}^{N}$, as described before. When 


\begin{tabular}{|c|c|}
\hline num_communities & The number of communities in the energy system \\
\hline width & Width of the grid (number of cells) where the communities are located \\
\hline height & Height of the grid (number of cells) where the communities are located \\
\hline num_rbfs & $\begin{array}{l}\text { Number of radial basis functions used for defining the functions that return the energy } \\
\text { produced by each energy resource at a given time step }\end{array}$ \\
\hline noise & $\begin{array}{l}\text { Positive value for introducing unpredictable variation in the amount of energy produced } \\
\text { by each energy resource (equation 5) }\end{array}$ \\
\hline min_capacity & The minimum storage capacity of any community in the system \\
\hline max_capacity & The maximum storage capacity of any community in the system \\
\hline min_storage_cost & The minimum unit cost for a community to store energy \\
\hline max_storage_cost & The maximum unit cost for a community to store energy \\
\hline neighbour_radius & Radius (number of cells) used to determine the neighbours of each community \\
\hline production_cost & The unit cost for the central energy system of producing energy \\
\hline purchasing_price & $\begin{array}{l}\text { The unit price for which the central energy system purchases energy from the commu- } \\
\text { nities }\end{array}$ \\
\hline selling_price & The unit price for which the central energy system sells energy to the communities \\
\hline$w$ & Weight of latest utility when updating the satisfaction of a community (equation 7) \\
\hline alpha & $\begin{array}{l}\text { Weight of the average satisfaction when calculating the performance of the system } \\
\text { (equation 8) }\end{array}$ \\
\hline beta & $\begin{array}{l}\text { Weight of the average proportion of unsatisfied communities when calculating the } \\
\text { performance of the system (equation } 8 \text { ) }\end{array}$ \\
\hline n_each_resource & $\begin{array}{l}\text { Number of resources of each kind (solar, wind, hydropower) possessed by each com- } \\
\text { munity that does have access to renewable energy resources }\end{array}$ \\
\hline perc_with_resources & Proportion of communities which possess renewable energy resources $((0,1])$ \\
\hline seed & Random seed (for obtaining reproducible results) \\
\hline
\end{tabular}

calculating the energy produced by each energy resource or the demand of a community at a given time step, we create a new set of weights, $\left\{w_{i}\right\}_{i=1}^{N}$, by adding a random perturbation to each $w_{i}^{\prime}$ which depends on the noise parameter:

$$
\begin{aligned}
& \sigma \sim \mathcal{U}(- \text { noise }, \text { noise }) \\
& w_{i}=w_{i}^{\prime}+\sigma
\end{aligned}
$$

The greater the value of noise, the greater the potential difference between $w_{i}$ and $w_{i}^{\prime}$ and, therefore, the greater the unpredictability of the functions which return the energy produced by each energy resource and the demand for each community. This model and particularly the functions simulating energy production are merely intuitive and make many simplifying assumptions. However, as we mentioned earlier in this section, realism has not been a major concern and the plausibility of the model we have created is satisfactory. It has enabled us to study the impact of adaptation and evolution of system policies.

Table 2 lists the input arguments in the model which have remained fixed in the experiments we have carried out in this paper. 


\begin{tabular}{|c|c|}
\hline num_communities & 50 \\
\hline width & 10 \\
\hline height & 10 \\
\hline num_rbfs & 10 \\
\hline min_capacity & 10 \\
\hline max_capacity & 30 \\
\hline min_storage_cost & 0.1 \\
\hline max_storage_cost & 0.3 \\
\hline neighbour_radius & 5 \\
\hline production_cost & 0.5 \\
\hline purchasing_price & 1 \\
\hline selling_price & 1.2 \\
\hline w & 0.5 \\
\hline alpha & 1 \\
\hline beta & 5 \\
\hline seed & 1995 \\
\hline
\end{tabular}

Table 2. Model input arguments fixed throughout the experiments

\section{REPRESENTATION AND APPLICATION OF POLICIES}

At every time step, each community's energy demand and the total energy which they have produced from their resources is calculated. The system's operating policy is then applied, returning a mode of operation which will determine what to do with the energy the communities have produced and how to meet their demands. In our model, the mode of operation has three degrees of freedom:

- What the communities should do with the energy they have produced at the current time step: either use it to satisfy their own demands (self-supply) or sell it all to the central system.

- In the case of self-supply, what the communities should do with any excess of energy: sell to the central system; store as much as capacity allows and sell the excess; trade it with neighbours and sell the excess; store, trade, and sell; or trade, store, and sell.

- If any demands have not been satisfied, the central system will ensure they are met by first reselling the energy which has been purchased from the communities and producing energy on demand (accounting for production costs) when necessary. The order in which the communities receive the energy is determined by several possible criteria: greatest demand, greatest production, lowest satisfaction, random, or ration.

Table 3 summarises these degrees of freedom and the values they can be set to when instantiating a mode of operation. For example, a possible mode of operation would be the triple \{SELF_SUPPLY, STORE + TRADE + SELL, GREATEST_DEMAND\}. There are therefore $2 \times 5 \times 5=50$ possible modes of operation which can be selected at each time step.

Regarding the first degree of freedom, if the value SELL is selected, then all the energy produced by each community at the current time step will be sold to the central system, the unit price being the input parameter purchasing_price. We make the simplifying assumption that the central system has a sufficiently large storage capacity and may therefore purchase all the energy which the communities have produced. If the value SELF_SUPPLY is selected, then the communities will try to meet their energy demands with their own energy assets. The energy assets of a community are the total energy it has available at the current time step: the energy produced by its resources and the energy it has stored. The communities use the energy they have produced and 


\begin{tabular}{|c|l|}
\hline Degree of Freedom & Possible Values \\
\hline Production & SELL, SELF_SUPPLY \\
\hline Excess & SELL, STORE + SELL, TRADE + SELL, STORE + TRADE + SELL, TRADE + STORE + \\
& SELL \\
\hline Redistribution & $\begin{array}{l}\text { GREATEST_DEMAND, GREATEST_PRODUCTION, LOWEST_SATISFACTION, RAN- } \\
\text { DOM, RATION }\end{array}$ \\
\hline
\end{tabular}

Table 3. Degrees of freedom and their possible values when instantiating a mode of operation

the energy stored, in this order, to cover their own demands; they may only use energy which has been stored after all the energy produced has been used.

When the communities are using a self-supply of energy, they may find themselves with an excess of energy produced if they are able to completely cover their own demands. This excess is handled according to the value of the second degree of freedom. The possible values represent the order in which the different options (selling to the central system, trading with other communities, or storing) should be tried. For example, the value STORE + TRADE + SELL means that communities should first attempt to store as much of the excess as their storage capacity allows; if there is still any energy left after storing, they will make it available for their neighbours to purchase (trade); and, if there is still any energy left after the trade, they will sell it to the central system. All possible values end with the option SELL because the communities may not be able to allocate all of their excess of energy by storing it or trading it with their neighbours. As before, the unit price of any energy sold to the central system is given by purchasing_price. Communities trade energy by using a simplified version of the Contract Net Protocol [9]; taking turns in random order, each community whose demand has not yet been satisfied attempts to purchase as much energy from its neighbours as possible, sorting them by the least selling price. When trading its excess of energy, each community has a unit selling price which is drawn from the uniform distribution $\mathcal{U}$ (selling_price/2, selling_price), where selling_price is the model input parameter which sets the unit price for which the central system sells energy to the communities (table 1). Storing energy has a unit cost associated, which, as mentioned in section 1 , is drawn from the uniform distribution $\mathcal{U}$ (min_storage_cost, max_storage_cost), where min_storage_cost and max_storage_cost are also input parameters of the model.

If the communities are not using a self-supply of energy or if there are communities whose demands have not been fully satisfied, then the central system will sell them energy. It starts by selling the energy it has stored, which has been previously purchased from the communities, for the unit price selling_price. If the energy in the storage is not enough, then the system will produce more energy. The selling price of this additional energy now increases according to a unit production cost given by the model input parameter production_cost. This means that, when this energy sale takes place, the last communities may have to purchase it for a higher unit price. The third degree of freedom of the mode of operation determines the order in which the communities purchase energy from the central system. They can be sorted by greatest demand, greatest production (both measured at the current time step), lowest satisfaction (which is cumulative, as explained ahead), or randomly. If the value RATION is selected, then the energy stored by the central system will be split equally among the communities; each community receives at most this ration and any additional demand is met with energy produced by the central system at a greater unit cost.

Applying the selected mode of operation at time step $t$ determines how much energy each community will produce for itself and how much it will sell, purchase, and store for the following time step. The resulting costs and revenues are taken into account when calculating the utility of this energy allocation for community $i$ at time step $t, u_{t}^{i}$ : 
Table 4. System variables collected at each time step

\begin{tabular}{|l|l|l|}
\hline Variable & Description & Value Range \\
\hline N & Number of communities (fixed throughout a model run) & $\mathbb{N}$ \\
\hline SAT & Mean satisfaction across all communities & $\mathbb{R}$ \\
\hline PROD & Total energy production across all communities & $\mathbb{R}^{+}$ \\
\hline SS & Total self-supply of energy across all communities & $\mathbb{R}_{0}^{+}$ \\
\hline MSSDD & Mean difference between self-supplied energy and demand across all communities & $\mathbb{R}$ \\
\hline MADD & $\begin{array}{l}\text { Mean difference between current assets (energy produced and stored) and demand } \\
\text { across all communities }\end{array}$ & $\mathbb{R}$ \\
\hline UNSAT & Number of unsatisfied agents (negative satisfaction) & $\mathbb{N}_{0}$ \\
\hline STO & Total energy stored across all communities & $\mathbb{R}_{0}^{+}$ \\
\hline DEM & Total demand across all communities & $\mathbb{R}^{+}$ \\
\hline TADD & $\begin{array}{l}\text { Total difference between current assets (energy produced and stored) and demand } \\
\text { across all communities }\end{array}$ & $\mathbb{R}$ \\
\hline MCL & $\begin{array}{l}\text { Mean capacity left across all communities (M is finite and the mean maximum } \\
\text { storage capacity across communities) }\end{array}$ & {$[0, M]$} \\
\hline GINI & Gini index of satisfaction inequality & $\mathbb{R}_{0}^{+}$ \\
\hline
\end{tabular}

$$
u_{t}^{i}=\text { total_sales }_{t}^{i}-\text { total_purchases }_{t}^{i}-\text { storage }_{-} \operatorname{costs}_{t}^{i}
$$

The cumulative satisfaction for community $i$ at time step $t$ is calculated with the most recent utility value as in equation 7 . The $w$ parameter weights the importance of past satisfactions and the current utility when updating a community's satisfaction.

$$
s_{t}^{i}=(1-w) \times s_{t-1}^{i}+w \times u_{t}^{i}, \text { with } s_{0}^{i}=0
$$

The operating policy which selects the mode of operation at each time step is represented by a triple of decision trees, one for each degree of freedom. The reason why we use decision trees is the need to evolve and adapt system policies using GP, which traditionally operates over tree structures. The inner nodes of the tree test the values of system-wide variables which are collected at each time step, returning a Boolean value (i.e., the decision trees are binary). Based on the literature about EIs, CPR management, and CESs, as well as on intuitive knowledge regarding the system model we have created, we have selected the system variables in table 4 to be collected and tested at each time step.

As a first step, we devised a default policy whose baseline performance could be compared to that of the evolved operating policies. As an example of the type of decision trees which are evolved and manipulated by our procedure, figures 2,3 , and 4 show the trees which make up the triple representing this default policy, selecting what the communities should do with the energy they have produced at each time step, what to do with any excess of energy in the case of self-supply, and the criteria under which the central system should redistribute energy, respectively. This default policy is the result of our intuition about the model we have created. Each function (inner) node of the decision trees could be any test on the values of system variables; we do not have a fixed set of function nodes. For this reason, they are represented internally as trees of operations. The root node of these trees is always a binary Boolean operator $(\leq,<,=, \neq,>, \geq)$, whereas all the other inner nodes are binary arithmetic operators $(+,-, \times)$. The leaf nodes are either the values of system-wide variables or real 


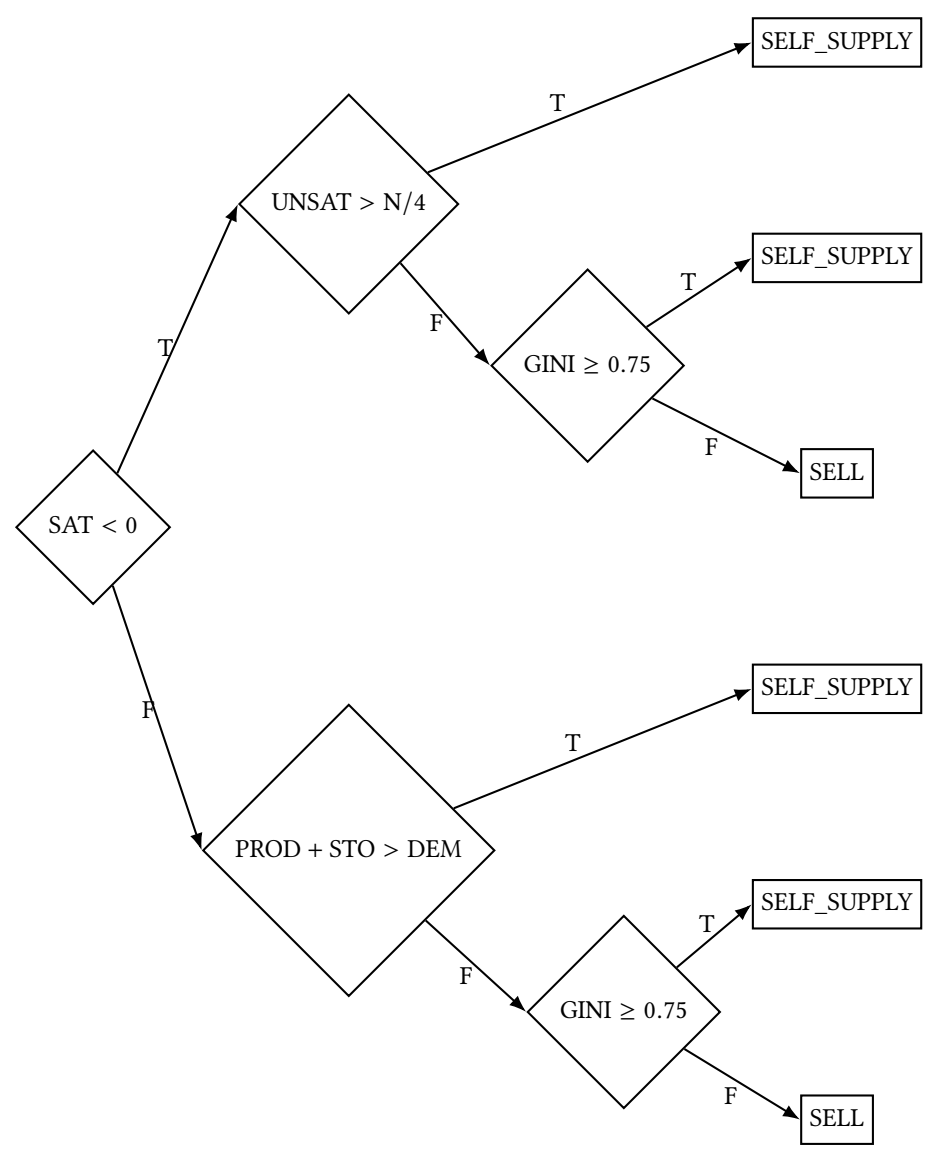

Fig. 2. Default decision tree encapsulating rules which determine what the communities should do with the energy they have produced at each time step

constants. This representation has enabled us to generate random function nodes when building new decision trees automatically with our optimisation procedure.

\section{OFFLINE PROCEDURE}

In order to find an optimal operating policy a priori, we have implemented a GP algorithm which evaluates alternatives by running the model with each of a population of policies for the number of time steps corresponding to a week (168, since time steps correspond to hours). A performance metric for each policy $i$ is calculated as shown in equation 8 , where satisfaction and the proportion of unsatisfied agents are averaged out after a week has passed. An agent is unsatisfied at a certain time step if its satisfaction is negative. The $\alpha$ parameter determines how much the average satisfaction favours the performance measure and the $\beta$ parameter determines how much the average proportion of unsatisfied agents penalises it $(\alpha>0$ and $\beta \geq 0)$. They are both input parameters of the model, as mentioned in section 1. 


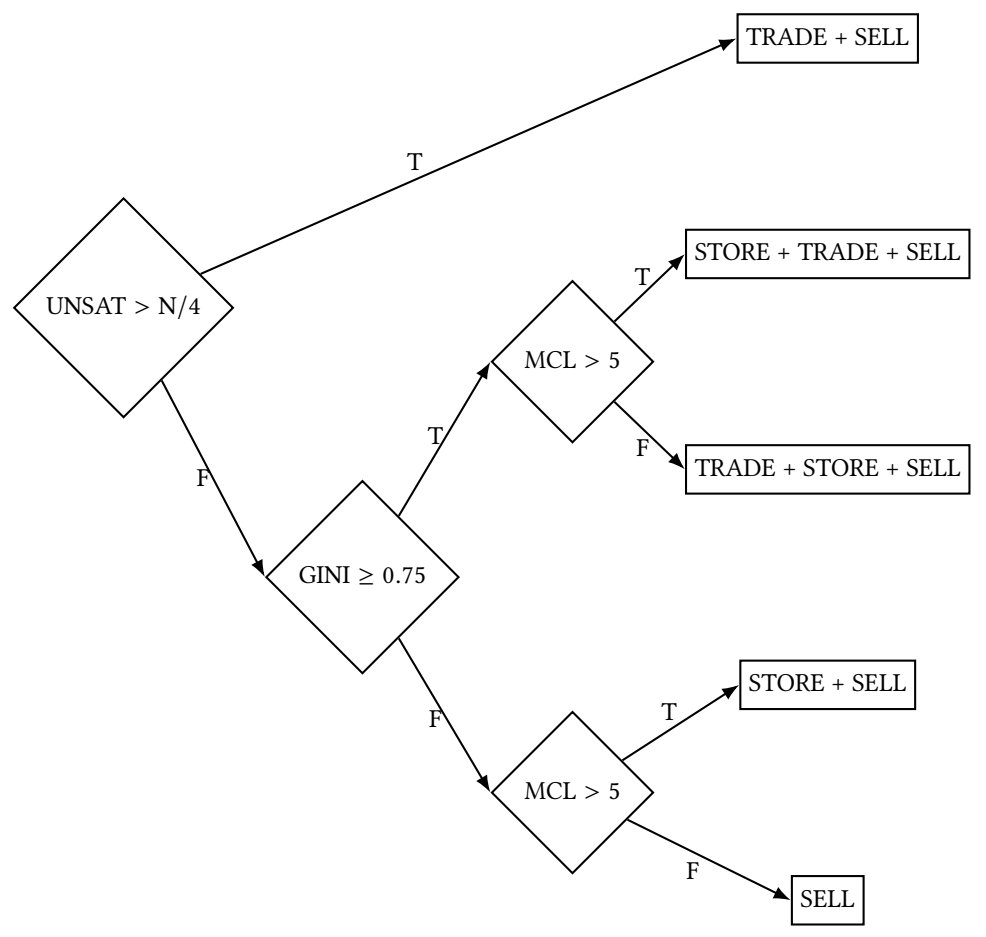

Fig. 3. Default decision tree encapsulating rules which determine what the communities should do at each time step with any excess of energy (in the case of self-supply)

$$
\begin{aligned}
\text { performance } & =\alpha \times \text { average satisfaction } \\
& -\beta \times \text { average proportion of unsatisfied agents }
\end{aligned}
$$

The procedure begins by randomly generating an initial population of operating policies (each a triple of decision trees, as explained before) using "ramped half and half" [7], with the possible values for each degree of freedom as leaf nodes. There is usually a fixed and finite set of function nodes in GP optimisation; however, as we have mentioned before, the set of possible function nodes for the decision trees is theoretically infinite in this case, as they can be any test on the values of system variables, so we randomly initialise a large set of these nodes at the start of the procedure. Since they are represented internally as trees of operations, we also use "ramped half and half" to generate them, with a fixed set of Boolean and arithmetic operators as inner nodes and the set of system variables as leaf nodes.

At each iteration of the optimisation procedure, the operating policies are evaluated by running instances of the same model for 168 time steps (a week) and computing a performance value, as in equation 8 . Running the model in order to obtain a performance value is a costly operation; for this reason, the alternative policies are tested on the model in parallel. The performance values are then used as fitness values to evolve a new generation using standard GP operations, namely reproduction, crossover, and mutation, which are described by Koza [7] Reproduction randomly selects individuals to be copied to the following generation with a probability which depends on their fitness value. If an elitist strategy is in place, then the policies with the highest fitness values are copied on to the following generation. The crossover operation randomly selects pairs of individuals, again 


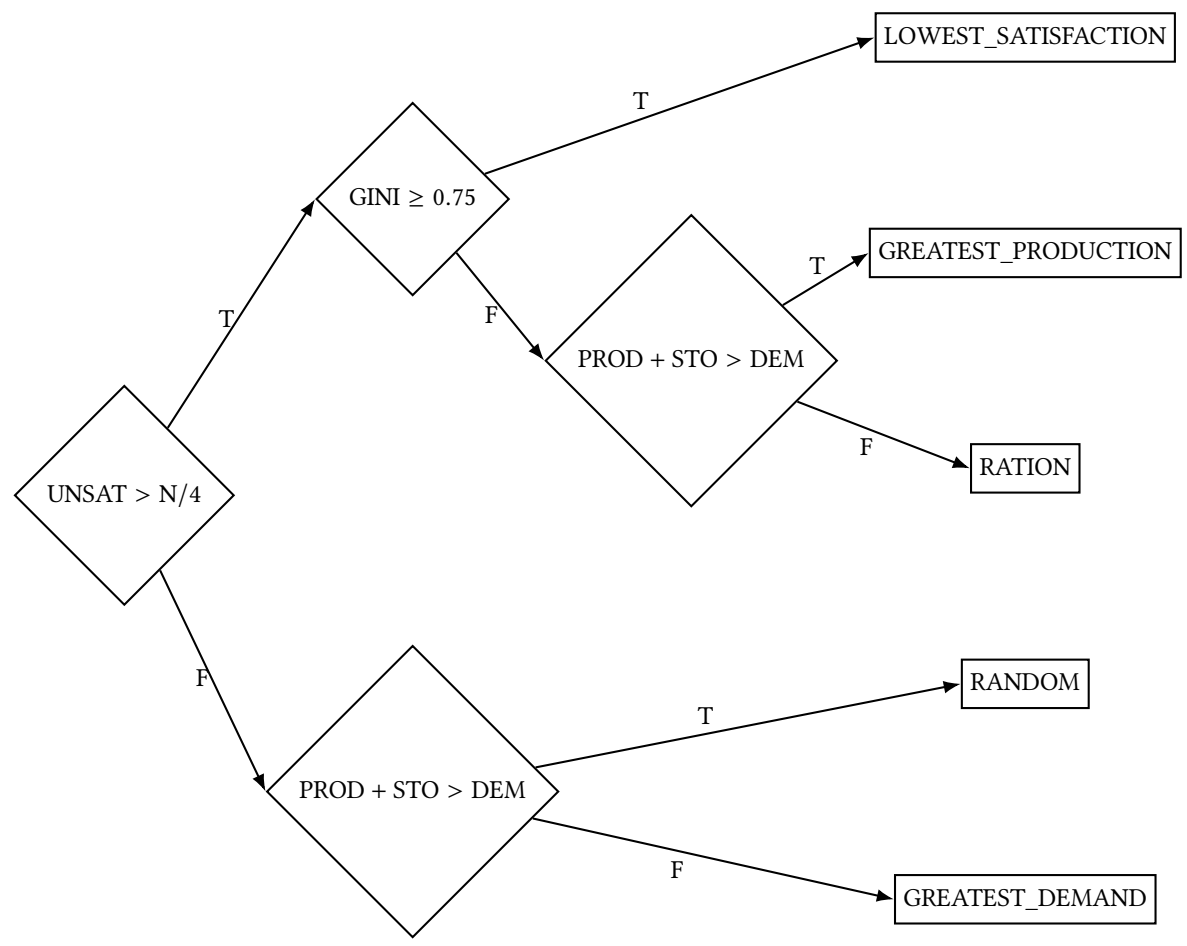

Fig. 4. Default decision tree encapsulating rules which determine how the central system redistributes energy

with a probability which is higher the higher their fitness, and crosses them element-wise, each element being a tree in the triple which makes up an operating policy. The mutation operation also selects individuals based on their fitness and creates new individuals by replacing parts of their trees with randomly generated subtrees; its goal is to introduce variability when searching for new solutions. For all of these operations, the probability of selecting a policy $i$ is a Softmax probability calculated with the fitness values of the policies after they have been evaluated on the system, as in equation 9. The reason why we have used Softmax probabilities is that they grow monotonically with the fitness values and this formulation handles negative fitness values elegantly.

$$
p_{i}=\frac{e^{\mathrm{fit}_{i}}}{\sum_{j} e^{\mathrm{fit}_{j}}}
$$

The offline procedure has some parameters which are summarised in table 5, which also reports the values they have been instantiated with in the experiments carried out in this paper. When generating the decision trees in the initial population, the procedure requires a parameter indicating their maximum depth, max_dt_depth_gen; the generated trees have depths ranging from 1 to max_dt_depth_gen (the root has depth 0 ). The "ramped half and half" method uses two approaches to generate trees, "grow" and "full". The parameter $n \_d t$ each sets the number of trees to be generated for each possible maximum depth (from 1 to max_dt_depth_gen) and each generation method ("grow" and "full"). As mentioned before, the function nodes in the decision trees are represented by trees of operations which are also generated using "ramped half and half"; $n \_o t \_e a c h$ and max_ot_depth_gen are the analogous parameters for generating these trees. When evolving a new generation, the crossover operation 


\begin{tabular}{|c|c|c|}
\hline Parameter & Description & Value used in the experiments \\
\hline n_dt_each & $\begin{array}{l}\text { Number of decision trees to be generated } \\
\text { for each tree depth and generation method } \\
\text { ("grow" and "full") using "ramped half and } \\
\text { half" }\end{array}$ & 3 \\
\hline max_dt_depth_gen & $\begin{array}{l}\text { Maximum depth for the decision trees gen- } \\
\text { erated using "ramped half and half" }\end{array}$ & 3 \\
\hline max_dt_depth & $\begin{array}{l}\text { Maximum depth for decision trees result- } \\
\text { ing from the crossover operation }\end{array}$ & 5 \\
\hline n_ot_each & $\begin{array}{l}\text { Number of trees of operations, which rep- } \\
\text { resent the function nodes in the decision } \\
\text { trees, to be generated for each tree depth } \\
\text { and generation method ("grow" and "full") } \\
\text { using "ramped half and half" }\end{array}$ & 20 \\
\hline max_ot_depth_gen & $\begin{array}{l}\text { Maximum depth for the trees of operations } \\
\text { generated using "ramped half and half" }\end{array}$ & 3 \\
\hline copy_perc & $\begin{array}{l}\text { Percentage of policies to be copied on to the } \\
\text { following generation (by either an elitist or } \\
\text { non-elitist strategy) }\end{array}$ & 0.1 \\
\hline cross_perc & $\begin{array}{l}\text { Percentage of policies in the following gen- } \\
\text { eration resulting from the crossover opera- } \\
\text { tion }\end{array}$ & 0.4 \\
\hline mut_perc & $\begin{array}{l}\text { Percentage of policies in the following gen- } \\
\text { eration resulting from the mutation opera- } \\
\text { tion }\end{array}$ & 0.5 \\
\hline elitist & $\begin{array}{l}\text { Boolean parameter indicating whether or } \\
\text { not to employ an elitist strategy when copy- } \\
\text { ing policies on to the following generation }\end{array}$ & False, copy individuals probabilistically \\
\hline
\end{tabular}

Table 5. Parameters of the offline procedure and their values throughout the experiments in this paper

may produce decision trees whose depth is larger than max_dt_depth_gen; the parameter max_dt_depth sets the maximum permissible depth for trees resulting from crossover to be included in the following generation.

The procedure keeps track of the best operating policy it has found so far and returns it after a certain number of generations have been evolved. This policy is the one which led to the greatest performance value after running the model, and, therefore, is approximately optimal. Algorithm 1 describes the procedure in pseudocode.

\section{REFERENCES}

[1] Aikaterini Bourazeri and Jeremy Pitt. 2014. An agent-based serious game for decentralised community energy systems. Lecture Notes in Computer Science 8861 (2014), 246-253. https://doi.org/10.1007/978-3-319-13191-7_20

[2] Scott Bucking and Vasken Dermardiros. 2018. Distributed evolutionary algorithm for co-optimization of building and district systems for early community energy masterplanning. Applied Soft Computing 63, Supplement C (2018), 14-22. https://doi.org/10.1016/j.asoc. 2017.10.044

[3] M D Buhmann and Jeremy Levesley. 2004. Radial Basis Functions: Theory and Implementations. Math. Comp. 73, 247 (2004), $1578-1581$. https://doi.org/10.1017/CBO9780511543241 


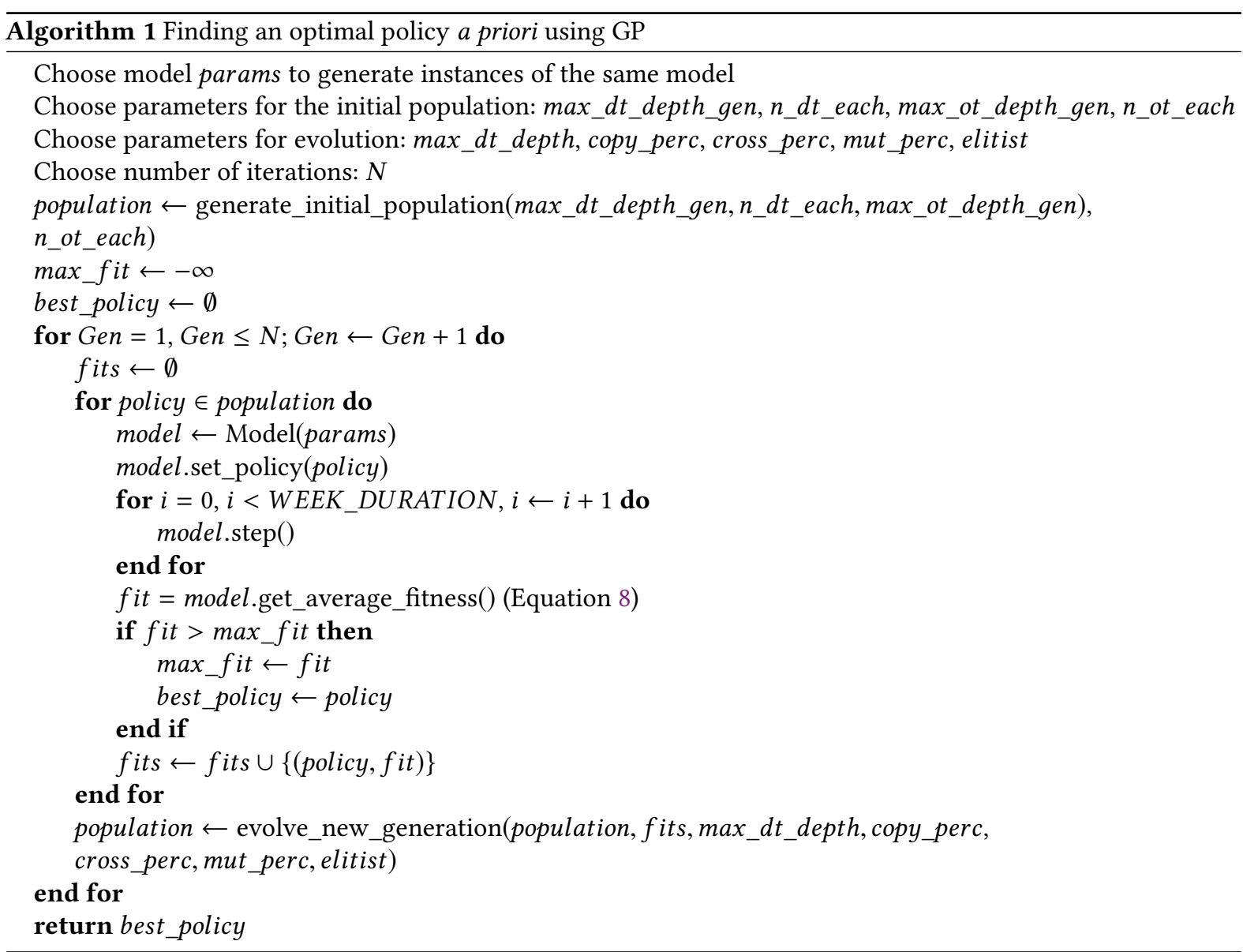

[4] J. W. Forrester. 1971. Counterintuitive Behavior of Social Systems. Simulation 16 (1971), 61-76. https://doi.org/10.1177/ 003754977101600202

[5] Zishuo Huang, Hang Yu, Zhenwei Peng, and Mei Zhao. 2015. Methods and tools for community energy planning: A review. , 1335-1348 pages. https://doi.org/10.1016/j.rser.2014.11.042

[6] Binod Prasad Koirala, Elta Koliou, Jonas Friege, Rudi A. Hakvoort, and Paulien M. Herder. 2016. Energetic communities for community energy: A review of key issues and trends shaping integrated community energy systems. , 722-744 pages. https://doi.org/10.1016/j rser.2015.11.080

[7] John R. Koza. 1992. Genetic Programming: On the Programming of Computers by Means of Natural Selection. MIT Press, Cambridge, MA, USA.

[8] Gonçalo Mendes, Christos Ioakimidis, and Paulo Ferrão. 2011. On the planning and analysis of Integrated Community Energy Systems: A review and survey of available tools. , 4836-4854 pages. https://doi.org/10.1016/j.rser.2011.07.067

[9] R. G. Smith. 1980. The Contract Net Protocol: High-Level Communication and Control in a Distributed Problem Solver. IEEE Trans. Comput. 29, 12 (Dec. 1980), 1104-1113. https://doi.org/10.1109/TC.1980.1675516

[10] Xiandong Xu, Xiaolong Jin, Hongjie Jia, Xiaodan Yu, and Kang Li. 2015. Hierarchical management for integrated community energy systems. Applied Energy 160 (2015), 231-243. https://doi.org/10.1016/j.apenergy.2015.08.134 